\title{
Electrocardiogram Signal Analysis - An Overview
}

\author{
Rajni \\ Associate Professor \\ SBSSTC, Ferozepur, Punjab
}

\author{
Inderbir Kaur \\ Research Scholar \\ SBSSTC, Ferozepur, Punjab
}

\begin{abstract}
Electrocardiogram (ECG) is the transthoracic interpretation of the electrical activity of the heart over a period of time. Analysis of ECG signal provides information regarding the condition of heart. Various methods like Fast Fourier Transforms, Wavelet Transform, etc. have been used for detection of cardiac diseases In this paper we have given a review on the work done in area of ECG signal analysis in past few years.
\end{abstract}

\section{General Terms}

Electrocardiogram, Heart

\section{Keywords}

Electrocardiogram(ECG), Wavelet Transforms

\section{INTRODUCTION}

Electrocardiogram is the electrical activity of the heart. It is a graphical demonstration of the variation of biopotential versus time [1]. The leads are placed on specific locations of the body of the person to record ECG either on graph paper or on monitors. The human heart contains four chambers i.e., Right Atrium, Left Atrium, Right Ventricle and Left Ventricle. The upper chambers are the two Atria's and the lower chambers are the two Ventricles. Under healthy condition the heartbeat begins at the Right Atrium called Sino Atria (SA) node and a special group of cells send these electrical signals across the heart. This signal travels from the Atria to the Atrio Ventricular (AV) node. The AV node connects to a group of fibers in Ventricles that conducts the electrical signal and transmits the impulse to all parts of the lower chamber, the Ventricles. To ensure that the heart is functioning properly this path of propagation must be traced accurately [2].The basic structure of heart is depicted in Figure.1:

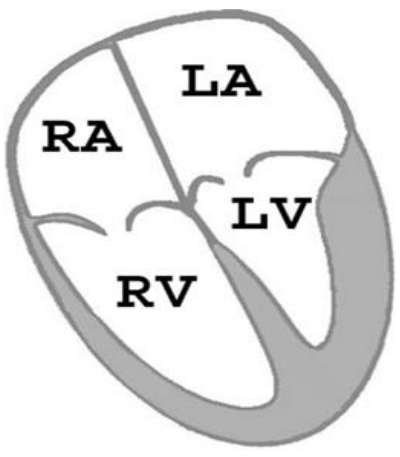

Figure 1. Schematic anatomy of the human heart [3]

\section{ECG WAVEFORM}

Each heart beat displayed is a sequence of electrical waves characterized by peaks and valleys. ECG mainly provides two kinds of information. One is the duration of the electrical wave passing through the heart and it will decide whether the electrical activity is normal or slow or irregular. Second is the amount of electrical activity passing through the heart muscle that helps to find whether the parts of the heart are too large or overworked. The frequency range of an ECG signal is 0.05$100 \mathrm{~Hz}$ and its dynamic range is $1-10 \mathrm{mV}$. The ECG signal is characterized by five peaks and valleys represented by the letters P, Q, R, S, T. Sometimes U wave is also present. The performance of ECG analysis is based on the accurate and reliable detection of the QRS complex as well as T- and $\mathrm{P}$ waves [4] [5]. An ideal ECG wave is as shown in Figure 2:

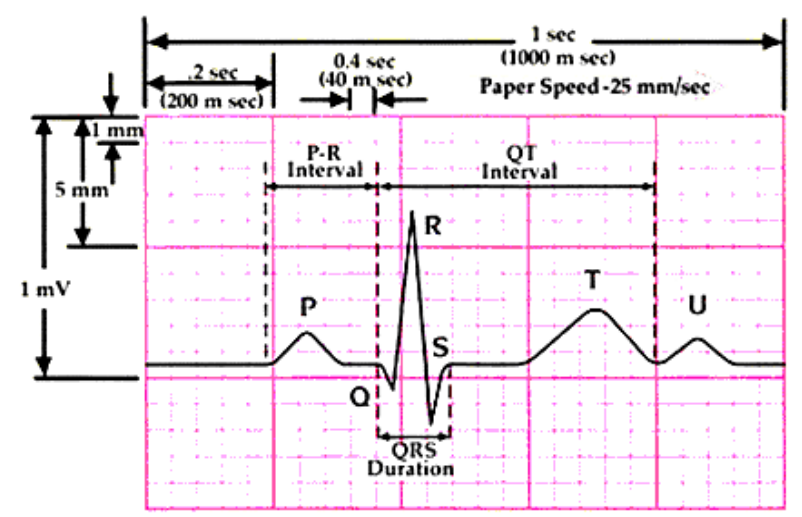

Figure2. A typical Cardiac Waveform [4]

The P-wave represent the activation of the two atria, the upper chambers of the heart, while the QRS complex and T-wave represent the excitation of the lower chamber of the heart, the ventricles. QRS detection is one of the fundamental issues in automatic ECG signal analysis. After QRS complex has been detected a thorough examination of ECG signal is done. The $\mathrm{P}$, QRS and T-waves reflect the rhythmic electrical depolarization and repolarization of the myocardium linked with the contractions of the atria and ventricles [6]. The horizontal section of this waveform prior to the P-wave is termed as the baseline or the isopotential line. The P-wave corresponds to the depolarization of the atrial musculature. The QRS complex gives the combined result of the repolarization of the atria and depolarization of the ventricles, which occurs almost at same time. The T-wave is the wave of ventricular repolarization, where as the $\mathrm{U}$-wave, if present is normally believed to be the result of after potentials in the ventricular muscle. So the duration amplitude and morphology of the QRS complex is helpful in diagnosing cardiac arrhythmias, conduction abnormalities, ventricular hypertrophy, myocardial infection and other disease states. The usual rate of heart is 60 to 100 beats per minute. A slower rate than the normal range is called bradycardia (slow heart) and a higher rate is called tachycardia (fast heart). If the ECG signal is not normal then an Arrhythmia is indicated [6] [7]. The waveform of normal ECG waveform and that of the abnormalities is shown in Figures 3, 4 and 5: 


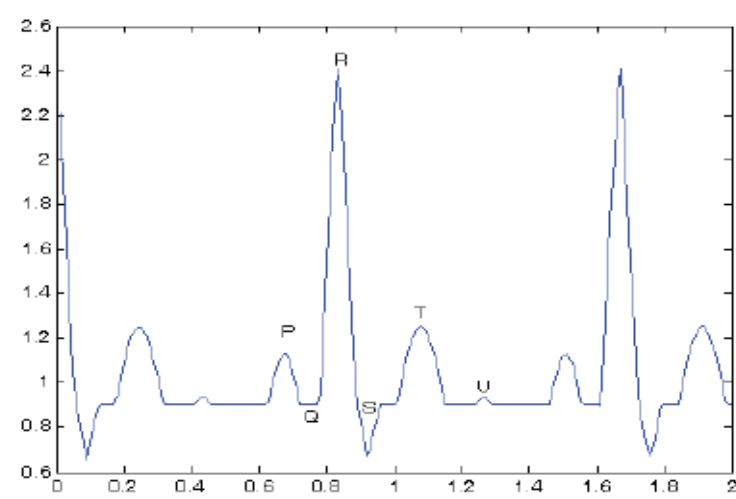

Figure3. Normal sinus rhythm [6]

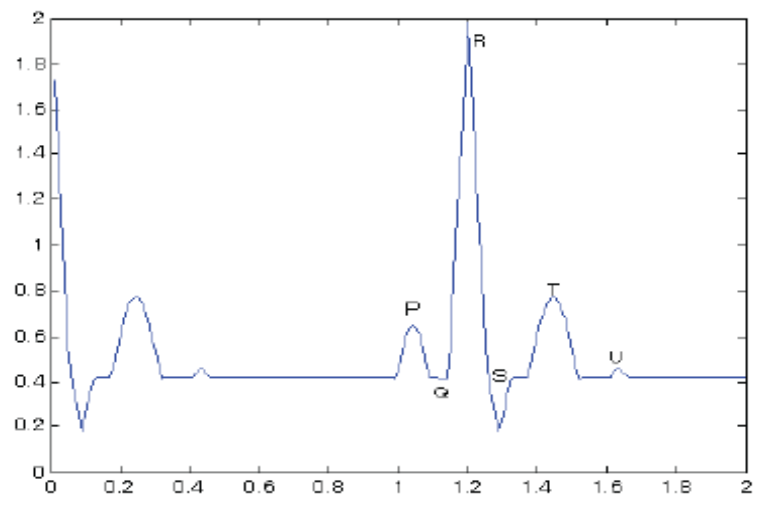

Figure 4. Sinus Bradycardia [6]

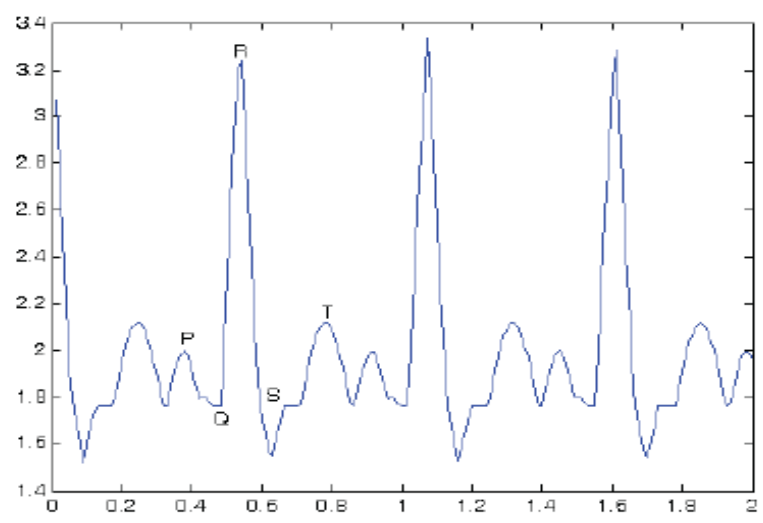

Figure 5. Sinus Tachycardia [6]

Various characteristic of the patterns of ECG wave are listed and the related disease to each pattern is also given [8]:

Table 1. Abnormalities

\begin{tabular}{|l|l|}
\hline ABNORMALITY & \multicolumn{1}{|c|}{ CHARACTERISTICS } \\
\hline Bradycardia & R-R interval $>1 \mathrm{~s}$ \\
\hline Tachycardia & R-R interval $<0.6 \mathrm{~s}$ \\
\hline Hypercalcaemia & QRS interval $<0.1 \mathrm{~s}$ \\
\hline
\end{tabular}

\begin{tabular}{|l|l|}
\hline Dextrocardia & Inverted P-wave \\
\hline Hyperkalemia & $\begin{array}{l}\text { Tall T-wave and absence of P- } \\
\text { wave }\end{array}$ \\
\hline $\begin{array}{l}\text { Sudden cardiac } \\
\text { death }\end{array}$ & Irregular ECG \\
\hline Sinoatrial block & $\begin{array}{l}\text { Complete drop out of a cardiac } \\
\text { cycle }\end{array}$ \\
\hline Myocardial ischemia & Inverted T-wave \\
\hline
\end{tabular}

\section{METHODS}

ECG signal analysis and detection has been an interesting topic from many years and still research is going on in this field. Various methods have been developed for ECG analysis as Fast Fourier transform, Short time Fourier transform, etc.

\subsection{Fast Fourier Transform (FFT)}

Earlier the method used for ECG signal analysis was time domain method. But the limitation was that it was not sufficient to study all characteristics of ECG signal [6] [9]. So a new method FFT was developed. Fourier transform is a well known method which transforms time domain signal to frequency domain to obtain the frequency coefficients [10]. FFT is an elementary transform in digital signal processing and has various applications in frequency analysis, signal processing etc [11]. It is a fast and more capable algorithm to work out the Discrete Fourier Transform (DFT) and obtains the same effect [12]. FFT is defined by the formula shown in equation (1):

$X \mathrm{k}=\sum_{n=0}^{N-1} x e^{-n k 2 \pi i / n}$

Where $\mathrm{k}$ is an integer ranging from 0 to $\mathrm{N}-1$ [13]. ECG signals can be compressed by using variety of techniques. One of the most important techniques is FFT. The total process consists of the following steps:

- Obtaining an ECG sample or input signal.

- Compressing the input signal by removing the low frequency components.

- Recovery of the original signal by using inverse FFT [14]

But the disadvantage of FFT is that it failed to provide the information regarding the accurate location of frequency components in time [1].

\subsection{Short Time Fourier Transform (STFT)}

To overcome this shortcoming of FFT, Dennis Gabor in 1946, first introduced the windowed-Fourier transform, i.e. ShortTime Fourier Transform known later as Gabor transform [15]. STFT has both time and frequency information [10]. It is used to determine the sinusoidal frequency and phase content of the signal as it varies with time. The STFT based spectrogram is a simple and fast technique in comparison to other timefrequency analysis. It is an easy approach of slicing the waveform of interest into a number of short-segments. Then it analyzes each segment using standard Fourier transform. A 
window function is applied to a segment of data, efficiently isolating that segment from the overall waveform, and Fourier transform is applied to that segment. This is known as the spectrogram or Short-Time Fourier Transform. For a signal x $(t)$, the definition of STFT is given by equation (2):

$$
X(\tau, f)=\int_{-T / 2}^{T / 2} x(t) w\left(t-\tau e^{-i 2 \pi f t}\right) d t
$$

Where $\mathrm{w}(\mathrm{t})$ is a window, having duration $\mathrm{T}$, centered at time location $t$, the Fourier transform of the windowed signal $\mathrm{x}(\mathrm{t}) \mathrm{w}(\mathrm{t}-\tau)$ is the STFT [16]. But the limitation of STFT is that its time frequency precision is not optimal. Hence a more suitable technique is opted to overcome this drawback.

\subsection{Wavelet Transform}

In STFT window should always have a fixed size and thus it does not give multi resolution information of the signal. But Wavelet Transform has the multiresolution property which gives both time and frequency information through variable window size [17]

In 1982 Jean Morlet a French geophysicist, introduced the concept of a 'Wavelet'. The Wavelet means a small wave and the study of Wavelet Transform is a new tool for seismic signal analysis. Without delay, Alex Grossmann theoretical physicists studied the inverse formula for the wavelet transform [15]. A Wavelet is a small wave which has energy concentrated in time and provides a tool for the analysis of transient, nonstationary or time-varying signals [18]

There are various Wavelets available to be used in large variety of applications. Wavelet families include Biorthogonal, Haar, Coiflet, Symlet, Daubechies Wavelets, etc. Some features which make them useful are:

- Wavelets are localized in both time and frequency

- For analyzing non-stationary signals such as ECG which have frequent level variations and uneven features.

- Wavelet separates a signal into multiresolution components [2].

The Wavelet Transform is a time-scale representation that has been used effectively in a variety of applications, in particular signal compression. It is a linear process that decomposes the signal into a number of scales associated with frequency components and analyzes each scale with a certain resolution [19]. Another advantage of Wavelet technique is various Wavelet functions available, that allows selecting the best function for analyzing the signal whereas in case of Fourier analysis it is restricted to one feature morphology that is the sinusoid [20].

Wavelet transforms can be classified into two categories:

- Continuous Wavelet Transforms (CWT)

- $\quad$ Discrete Wavelet Transforms (DWT)

\subsubsection{Continuous Wavelet Transform}

The CWT is a time-frequency analysis method which differs from the traditional STFT since it allows high localization in time of high frequency signal features. The CWT does this by having a variable window width, which is associated to the scale of observation - a flexibility that allows for the isolation of the high frequency features. Another important difference from the STFT is that the CWT is not restricted to use sinusoidal analyzing functions. Rather, localized waveforms can be selected as long as they satisfy the predefined mathematical criteria [20].

The CWT of a signal $\mathrm{x}(\mathrm{t})$ is defined as:

$$
W(a, b)=1 / \sqrt{ } a \int_{-\infty}^{\infty} f(t) h *((t-b) / a) d t
$$

$\mathrm{h}(\mathrm{t})$ is called mother wavelet, $\mathrm{a}$ is the scaling parameter in $\mathrm{y}$ axis and $\mathrm{b}$ is the shift parameter in $\mathrm{X}$-axis [2].

\subsubsection{Discrete Wavelet Transform}

The DWT is defined as:

$$
W(j, k)=\sum_{j} \sum_{k} x(k) e^{-\frac{j}{2}} \psi\left(2^{-j} n-k\right)
$$

Where $\Psi(t)$ is a time function with finite energy and fast decay called the mother wavelet [1].

\section{PERFORMANCE ANALYSIS}

It is expressed by following equations:

Sensitivity $(\mathrm{Se})=\mathrm{TP} /(\mathrm{TP}+\mathrm{FN})$

Positive predictivity $(\mathrm{P}+)=\mathrm{TP} /(\mathrm{TP}+\mathrm{FP})$

TP=True Positive, FN=False Negative, FP=False Positive

\section{CONCLUSION}

This paper gives a review on various techniques used to detect abnormalities in ECG signal. Wavelet Transform is the flexible tool which can be used for signal analysis. In future more research could be done on Wavelet Transform to be applied to ECG.

\section{REFERENCES}

[1] Apoorv Gautam and Maninder Kaur, "ECG Analysis using Continuous Wavelet Transforms (CWT)", IOSR Journal of Engineering, Apr.2012, Vol.2 (4), 632-635.

[2] Nagendra H, S. Mukherjee and Vinod Kumar, "Application of Wavelet Techniques in ECG Signal Processing: An Overview", International Journal of Engineering Science and Technology (IJEST), October 2011, Vol.3, No.10, 7432-7443

[3] Adam Josko and Remigiusz J. Rak, "Effective Simulation of Signals for Testing ECG Analyzer", IEEE Transactions on Instrumentation and Measurement, June 2005, Vol.54, No.3, 1019-1024.

[4] K.V.L. Narayana, A. Bhujanga Rao," Wavelet based QRS detection in ECG using MATLAB", Innovative Systems Design and Engineering, 2011, Vol. 2, No 7, 6069.

[5] B. Anuradha, K. Suresh Kumar and V.C. Veera Reddy, "Classification of Cardiac signals using Time Domain Methods", ARPN Journal of Engineering and Applied Sciences, June 2008, Vol.3, No.3, 7-12.

[6] C. Saritha, V. Sukanya, and Y. Narsimha Murthy," ECG Signal Analysis Using Wavelet Transforms", Bulg.J.Phys.35, 2008, 68-77.

[7] Rajiv Ranjan, V.K Giri, "A Unified Approach of ECG Signal Analysis", International Journal of Soft Computing and Engineering (IJSCE), July 2012, Volume-2, Issue-3, 5-10. 
[8] A. Muthuchudar, Lt. Dr. S. Santosh Baboo, "A Study of the Processes Involved in ECG Signal Analysis", International Journal of Scientific and Research Publications, March 2013, Volume 3, Issue 3, 1-5.

[9] S. Karpagachelvi, Dr. M. Arthanari, M. Sivakumar, "ECG Extraction Techniques- A Survey Approach", International Journal of Computer Science and Information Security, April 2010, Vol .8.No .1, 76-80.

[10] A.K.M Fazlul Haque, "Improved Detection of ECG Features Using Wavelet for Emergency Medical Application", IJAITI, Mar/Apr 2012, Vol. 1, No. 2.

[11] Pushpendra Singh, Om Prakash Yadav, Yojana Yadav, "ECG Signal Compression Implementation by a New 2Dimensional Transform Technique", International Journal of Engineering and Advanced Technology (IJEAT), August 2012, Vol.1, Issue-6.

[12] Vikramaditya Dangi, Amol Parab and Kshitij Pawar, 2012. Analysis of Transform Based ECG Compression Techniques. Proc. of the International Conference on Advances in Electronics and Electrical Engineering AEEE.

[13] Dr. Anup Mishra, Nisha Deshmukh, Aarti Verma," Compression of ECG Signal Using Fast Fourier Transform Technique-A Survey Approach", International Journal of Engineering Research \& Technology (IJERT), August,2013, Vol.2,Issue 8.

[14] Bishweshwar Pratap Tasa, Pompy Das, Avinash Sinha, Tulika Chenglari, Hemashree Bordoloi," Simulation
Based R-peak and QRS complex detection in ECG Signal", Current Trends in Technology and Science, 2013, Volume 2, Issue 4.

[15] M. Sifuzzaman, M.R. Islam , M.Z. Ali," Application of Wavelet Transform and its Advantages Compared to Fourier Transform”, Journal of Physical Sciences, 2009, Vol. 13,121-134.

[16] K. Rawal, B.S. Saini and D. Singh, 6-7 December 2012. Data Fusion of Time Frequency Analysis methods with application to Biomedical Signals. $2^{\text {nd }}$ International Conference on Biomedical Engineering \& Assistive Technologies, 173-177.

[17] P. Karthikeyan, M. Murugappan, and S. Yaacob," ECG Signal Denoising Using Wavelet Thresholding Techniques in Human Stress Assessment", International Journal on Electrical Engineering and Informatics , July 2012, Volume 4, Number 2.

[18] Mikhled Alfouri and Khaled Daqrouq, "ECG Signal Denoising by Wavelet Transform Thresholding", American Journal of Applied Sciences 5(3), 2008, 276281.

[19] Priyanka Mehta, Monika Kumari," QRS Complex Detection of ECG Signal Using Wavelet Transform", International Journal of Applied Engineering Research, 2012, Vol.7 No.11.

[20] Paul S Addison," Wavelet Transforms and the ECG: a review", Institute of Physics Publishing, Physiol. Meas. 26, 2005, R155-R199. 